\title{
Hereditary right Jacobson radicals of type-1(e) and 2(e) for right near-rings
}

\author{
Ravi Srinivasa Rao and K. Siva Prasad
}

\begin{abstract}
Near-rings considered are right near-rings. In this paper two more radicals, the right Jacobson radicals of type-1(e) and 2(e), are introduced for near-rings. It is shown that they are Kurosh-Amitsur radicals (KAradicals) in the class of all near-rings and are ideal-hereditary radicals in the class of all zero-symmetric near-rings. Different kinds of examples are also presented.
\end{abstract}

\section{Introduction}

Near-rings considered are right near-rings and not necessarily zero-symmetric, and $\mathrm{R}$ is a near-ring. The (left) Jacobson radicals $\mathrm{J}_{2(0)}$ and $\mathrm{J}_{3(0)}$ introduced by Veldsman [14] and the (right) Jacobson radical $\mathrm{J}_{0(e)}^{r}$ introduced by the authors with T. Srinivas [13] are the only known Jacobson-type radicals which are Kurosh-Amitsur in the class of all near-rings and ideal-hereditary in the class of all zero-symmetric near-rings. It is also known that (Corollary 6 of [15]) there is no non-trivial ideal-hereditary radical in the class of all nearrings.

In [5] and [6] the first author has shown that as in rings, matrix units determined by right ideals identify matrix near-rings. The importance of the right Jacobson radicals of type- $\nu, \nu \in\{0,1,2, \mathrm{~s}\}$ of near-rings introduced by the authors in [7], [8] and [9], in the extension of a form of the Wedderburn-Artin

Key Words: Right R-groups of type-1(e) and 2(e), right primitive ideals of type-1(e) and $2(e)$, right Jacobson radicals of type-1(e) and $2(e)$, KA-radicals, hereditary radicals.

2010 Mathematics Subject Classification: Primary 16Y30

Received: August, 2011.

Accepted: April, 2012 
theorem of rings involving the matrix rings to near-rings, is established in [12]. In [10] and [11] the authors with T. Srinivas have shown that the right Jacobson radicals of type- 0,1 and 2 are Kurosh-Amitsur radicals (KA-radicals) in the class of all zero-symmetric near-rings but they are not ideal-hereditary in that class.

In this paper right $\mathrm{R}$-groups of type- $\nu(\mathrm{e})$, right $\nu(\mathrm{e})$-primitive ideals and right $\nu(\mathrm{e})$-primitive near-rings are introduced, $\nu \in\{1,2\}$. Using them the right Jacobson radical of type- $\nu(\mathrm{e})$ is introduced for near-rings and is denoted by $\mathrm{J}_{\nu(e)}^{r}, \nu \in\{1,2\}$. A right $\nu(\mathrm{e})$-primitive ideal of $\mathrm{R}$ is an equiprime ideal of $\mathrm{R}$. It is shown that $\mathrm{J}_{\nu(e)}^{r}$ is a Kurosh-Amitsur radical in the class of all near-rings and is an ideal-hereditary radical in the class of all zero-symmetric near-rings, $\nu \in\{1,2\}$. Moreover, for any ideal I of $\mathrm{R}, \mathrm{J}_{\nu(e)}^{r}(\mathrm{I}) \subseteq \mathrm{J}_{\nu(e)}^{r}(\mathrm{R}) \cap \mathrm{I}$ with equality, if $\mathrm{I}$ is left invariant, $\nu \in\{1,2\}$.

\section{Preliminaries}

Near-rings considered are right near-rings and not necessarily zero-symmetric. Unless otherwise specified $\mathrm{R}$ stands for a right near-ring. Near-ring notions not defined here can be found in Pilz [4].

$\mathrm{R}_{0}$ and $\mathrm{R}_{c}$ denotes the zero-symmetric part and constant part of $\mathrm{R}$ respectively. Now we give here some definitions of [7] and [8].

A group $(\mathrm{G},+)$ is called a right $R$-group if there is a mapping $((\mathrm{g}, \mathrm{r}) \rightarrow \mathrm{gr})$ of $\mathrm{G} \times \mathrm{R}$ into $\mathrm{G}$ such that $(1)(\mathrm{g}+\mathrm{h}) \mathrm{r}=\mathrm{gr}+\mathrm{hr},(2) \mathrm{g}(\mathrm{rs})=(\mathrm{gr}) \mathrm{s}$, for all $\mathrm{g}$, $\mathrm{h} \in \mathrm{G}$ and $\mathrm{r}, \mathrm{s} \in \mathrm{R}$. A subgroup (normal subgroup) $\mathrm{H}$ of a right $\mathrm{R}$-group $\mathrm{G}$ is called an $R$-subgroup (ideal) of $\mathrm{G}$ if $\mathrm{hr} \in \mathrm{H}$ for all $\mathrm{h} \in \mathrm{H}$ and $\mathrm{r} \in \mathrm{R}$.

Let $\mathrm{G}$ be a right R-group. An element $g_{0} \in G$ is called a generator of $\mathrm{G}$ if $g_{0} R=G$ and $g_{0}(r+s)=g_{0} r+g_{0} s$ for all $\mathrm{r}, \mathrm{s} \in \mathrm{R}$. $\mathrm{G}$ is said to be monogenic if $\mathrm{G}$ has a generator. $\mathrm{G}$ is said to be simple if $\mathrm{G} \neq\{0\}$ and $\mathrm{G}$, and $\{0\}$ are the only ideals of $\mathrm{G}$.

A monogenic right $\mathrm{R}$-group $\mathrm{G}$ is said to be a right $R$-group of type- 0 if $\mathrm{G}$ is simple.

The annihilator of $\mathrm{G}$ denoted by $(0: \mathrm{G})$ is defined as $(0: \mathrm{G})=\{\mathrm{a} \in \mathrm{R} \mid \mathrm{Ga}$ $=\{0\}\}$.

A right $\mathrm{R}$-group $\mathrm{G}$ of type- 0 is said to be of type-1 if $\mathrm{G}$ has exactly two $\mathrm{R}$ subgroups, namely $\{0\}$ and $\mathrm{G}$.

A right R-group $\mathrm{G}$ of type- 0 is said to be of type-2 if $\mathrm{gR}=\mathrm{G}$ for all $g \in G \backslash\{0\}$. Note that a right R-group of type- 2 is of type- 1 and a right R-group of type- 1 is of type- 0 .

Let $\nu \in\{0,1,2\}$. A right modular right ideal $\mathrm{K}$ of $\mathrm{R}$ is called right $\nu$-modular if $\mathrm{R} / \mathrm{K}$ is a right $\mathrm{R}$-group of type- $\nu$.

An ideal $\mathrm{P}$ of $\mathrm{R}$ is called right $\nu$-primitive if $\mathrm{P}$ is the largest ideal of $\mathrm{R}$ contained 
in a right $\nu$-modular right ideal of $\mathrm{R}$. $\mathrm{R}$ is called a right $\nu$-primitive near-ring if $\{0\}$ is a right $\nu$-primitive ideal of $\mathrm{R}$.

$J_{\nu}^{r}(R)$ denotes the intersection of all right $\nu$-primitive ideals of $\mathrm{R}$. If $\mathrm{R}$ has no right $\nu$-primitive ideals, then $\mathrm{J}_{\nu}^{r}(\mathrm{R})$ is defined as $\mathrm{R}$. $\mathrm{J}_{\nu}^{r}$ is called the right Jacobson radical of type- $\nu$.

A near-ring $\mathrm{R}$ is called an equiprime near-ring ([1]) if $0 \neq \mathrm{a} \in \mathrm{R}, \mathrm{x}, \mathrm{y} \in \mathrm{R}$ and arx $=$ ary for all $\mathrm{r} \in \mathrm{R}$, implies $\mathrm{x}=\mathrm{y}$. An ideal $\mathrm{I}$ of $\mathrm{R}$ is called equiprime if $\mathrm{R} / \mathrm{I}$ is an equiprime near-ring.

It is known that a near-ring $\mathrm{R}$ is equiprime if and only if ([1])

1. $\mathrm{x}, \mathrm{y} \in \mathrm{R}$ and $\mathrm{xRy}=\{0\}$ implies $\mathrm{x}=0$ or $\mathrm{y}=0$.

2. If $\{0\} \neq \mathrm{I}$ is an invariant subnear-ring of $\mathrm{R}, \mathrm{x}, \mathrm{y} \in \mathrm{R}$ and $\mathrm{ax}=$ ay for all a $\in \mathrm{I}$ implies $\mathrm{x}=\mathrm{y}$.

Moreover, an equiprime near-ring is zero-symmetric.

If $\mathrm{I}$ is an ideal of $\mathrm{R}$, then we denote it by $\mathrm{I} \triangleleft \mathrm{R}$. A subset $\mathrm{S}$ of $\mathrm{R}$ is left invariant if RS $\subseteq \mathrm{S}$. By a radical class we mean a radical class in the sense of KuroshAmitsur. Let $\mathcal{E}$ be a class of near-rings. $\mathcal{E}$ is called regular if $\{0\} \neq \mathrm{I} \triangleleft \mathrm{R} \in \mathcal{E}$ implies that $\{0\} \neq \mathrm{I} / \mathrm{K} \in \mathcal{E}$ for some $\mathrm{K} \triangleleft \mathrm{I}$. A class $\mathcal{E}$ is called hereditary if $\mathrm{I} \triangleleft \mathrm{R} \in \mathcal{E}$ implies $\mathrm{I} \in \mathcal{E}$. $\mathcal{E}$ is called c-hereditary if $\mathrm{I}$ is a left invariant ideal of $\mathrm{R} \in \mathcal{E}$ implies $\mathrm{I} \in \mathcal{E}$. It is clear that a hereditary class is a regular class. If $\mathrm{I} \triangleleft \mathrm{R}$ and for every non zero ideal $\mathrm{J}$ of $\mathrm{R}, \mathrm{J} \cap \mathrm{I} \neq\{0\}$, then $\mathrm{I}$ is called an essential ideal of $\mathrm{R}$ and is denoted by $\mathrm{I} \triangleleft$. R. A class of near-rings $\mathcal{E}$ is called closed under essential extensions (essential left invariant extensions) if $\mathrm{I} \in \mathcal{E}$, $\mathrm{I} \triangleleft \cdot \mathrm{R}$ ( $\mathrm{I}$ is an essential ideal of $\mathrm{R}$ which is left invariant) implies $\mathrm{R} \in \mathcal{E}$. A class of near-rings $\mathcal{E}$ is said to satisfy condition $\left(F_{l}\right)$ whenever $\mathrm{K} \triangleleft \mathrm{I} \triangleleft \mathrm{R}$, and $\mathrm{I}$ is left invariant in $\mathrm{R}$ and $\mathrm{I} / \mathrm{K} \in \mathcal{E}$, it follows that $\mathrm{K} \triangleleft \mathrm{R}$.

In [2], G. L. Booth and N. J. Groenewald defined special radicals for nearrings. A class $\mathcal{E}$ consisting of equiprime near-rings is called a special class if it is hereditary and closed under left invariant essential extensions. If $\mathcal{R}$ is the upper radical in the class of all near-rings determined by a special class of near-rings, then $\mathcal{R}$ is called a special radical. If $\mathcal{R}$ is a radical class, then the class $\mathcal{S} \mathcal{R}=\{\mathrm{R} \mid \mathcal{R}(\mathrm{R})=\{0\}\}$ is called the semisimple class of $\mathcal{R}$.

We also need the following Theorem:

Theorem 2.1. (Theorem 2.4 of [14]) Let $\mathcal{E}$ be a class of zero-symmetric near-rings. If $\mathcal{E}$ is regular, closed under essential left invariant extensions and satisfies condition $\left(F_{l}\right)$, then $\mathcal{R}:=\mathcal{U} \mathcal{E}$ is a c-hereditary radical class in the variety of all near-rings, $\mathcal{S} \mathcal{R}=\overline{\mathcal{E}}$ and $\mathcal{S} \mathcal{R}$ is hereditary. So, $\mathcal{R}(R)=\cap\{I \triangleleft R$ $\mid R / I \in \mathcal{E}\}$ for any near-ring $R$.

Remark 2.2. Since all ideals in a zero-symmetric near-ring are left invariant, under the hypothesis of Theorem 2.1, in the variety of zero-symmetric nearrings both $\mathcal{R}$ and $\mathcal{S} \mathcal{R}$ are hereditary and hence the radical is ideal-hereditary, 
that is, if $\mathrm{I} \triangleleft \mathrm{R}$, then $\mathcal{R}(\mathrm{I})=\mathrm{I} \cap \mathcal{R}(\mathrm{R})$.

Proposition 2.3. (Proposition 3.3 of [1]) The class of all equiprime nearrings is closed under essential left invariant extensions.

Proposition 2.4. (Corollary 2.4 of [1]) The class of all equiprime near-rings satisfies condition $\left(F_{l}\right)$.

We need the following results of [11].

Theorem 2.5. (Theorems 3.1 and 3.2 of [11]) Let $G$ be a right $R$-group of type- $\nu, \nu \in\{1,2\}$. If $S$ is an invariant subnear-ring of $R$ and $G S \neq\{0\}$, then $G$ is also a right $S$-group of type- $\nu$.

Theorem 2.6. (Theorems 3.9 and 3.11 of [11]) Let $S$ be an invariant subnear-ring of $R$. If $G$ is a right $S$-group of type- $\nu, \nu \in\{1,2\}$, then $G$ is a right $R$-group of type- $\nu$.

\section{The right Jacobson radical of type- $\nu(e), \nu \in\{1,2\}$.}

Throughout this section $\nu \in\{1,2\}$. In this section first we introduce right R-groups of type- $\nu(e)$ and study some of their properties. Using them we introduce right Jacobson radical of type- $\nu(e)$ and study its properties.

We begin with some basic properties of right R-groups of type- $\nu$.

The following Proposition is proved in [11] (Corollary 3.4).

We give here a different proof.

Proposition 3.1. Let $G$ be a right $R$-group of type- $\nu$. Then $G R_{c}=\{0\}$.

Proof. Let $\mathrm{g}_{0}$ be a generator of $\mathrm{G}$. So $\mathrm{g}_{0}$ is distributive over $\mathrm{R}$, that is, $\mathrm{g}_{0}(\mathrm{r}+$ $\mathrm{s})=\mathrm{g}_{0} \mathrm{r}+\mathrm{g}_{0} \mathrm{~s}$ for all $\mathrm{r}, \mathrm{s} \in \mathrm{R}$ and $\mathrm{g}_{0} \mathrm{R}=\mathrm{G}$. Since $\mathrm{g}_{0}$ is distributive over $\mathrm{R}$ and $\mathrm{R}_{c}$ is an $\mathrm{R}$-subgroup of the right $\mathrm{R}$-group $\mathrm{R}, \mathrm{g}_{0} \mathrm{R}_{c}$ is an $\mathrm{R}$-subgroup of the right $\mathrm{R}$-group $\mathrm{G}$. Also since $\mathrm{G}$ has no nontrivial right $\mathrm{R}$-subgroups, $\mathrm{g}_{0} \mathrm{R}_{c}=\{0\}$ or G. If $\mathrm{g}_{0} \mathrm{R}_{c}=\mathrm{G}$, then $\mathrm{g}_{0} \mathrm{r}_{c}=\mathrm{g}_{0}$ for some $\mathrm{r}_{c} \in \mathrm{R}_{c}$. Therefore, $\mathrm{g}_{0} \mathrm{x}=\left(\mathrm{g}_{0} \mathrm{r}_{c}\right) \mathrm{x}$ $=\mathrm{g}_{0}\left(\mathrm{r}_{c} \mathrm{x}\right)=\mathrm{g}_{0} \mathrm{r}_{c}=\mathrm{g}_{0}$ for all $\mathrm{x} \in \mathrm{R}$. So $\mathrm{G}=\mathrm{g}_{0} \mathrm{R}=\left\{\mathrm{g}_{0}\right\}$, a contradiction. Hence, $\mathrm{g}_{0} \mathrm{R}_{c}=\{0\}$. Let $\mathrm{g} \in \mathrm{G}$. We have $\mathrm{g}=\mathrm{g}_{0}$ s for some $\mathrm{s} \in \mathrm{R}$. Now $\operatorname{gr}_{c}=$ $\left(\mathrm{g}_{0} \mathrm{~s}\right) \mathrm{r}_{c}=\mathrm{g}_{0}\left(\mathrm{sr}_{c}\right)=0$, as $\mathrm{sr}_{c} \in \mathrm{R}_{c}$. So, $\mathrm{GR}_{c}=\{0\}$.

The following Proposition follows from Proposition 3.7 of [13].

Proposition 3.2. Let $G$ be a right $R$-group of type- $\nu$. Then there is a largest ideal of $R$ contained in $(0: G)=\{r \in R \mid G r=\{0\}\}$. 
Definition 3.3. Let $\mathrm{G}$ be a right R-group of type- $\nu$. Suppose that $\mathrm{P}$ is the largest ideal of $\mathrm{R}$ contained in $(0: \mathrm{G})=\{\mathrm{r} \in \mathrm{R} \mid \mathrm{Gr}=\{0\}\}$. Then $\mathrm{G}$ is said to be a right R-group of type- $\nu(e)$ if $0 \neq \mathrm{g} \in \mathrm{G}, \mathrm{r}_{1}, \mathrm{r}_{2} \in \mathrm{R}$ and $\operatorname{gxr}_{1}=\operatorname{gxr}_{2}$ for all $\mathrm{x} \in \mathrm{R}$ implies $\mathrm{r}_{1}-\mathrm{r}_{2} \in \mathrm{P}$.

Proposition 3.4. Let $G$ be a right $R$-group of type- $\nu$. Let $P$ be the largest ideal of $R$ contained in $(0: G)$. Then the following are equivalent.

1. $G$ is a right $R$-group of type- $\nu(e)$.

2. $r_{1}, r_{2} \in R$ and $g r_{1}=g r_{2}$ for all $g \in G$ implies $r_{1}-r_{2} \in P$.

Proof. Let $\mathrm{g}_{0}$ be a generator of the right R-group G. (1) implies (2) follows from the definition of a right R-group of type- $\nu(\mathrm{e})$ as $\mathrm{g}_{0} \mathrm{R}=\mathrm{G}$. Assume (2). Suppose that $0 \neq \mathrm{g} \in \mathrm{G}, \mathrm{r}_{1}, \mathrm{r}_{2} \in \mathrm{R}$ and $\operatorname{gxr}_{1}=\operatorname{gxr}_{2}$ for all $\mathrm{x} \in \mathrm{R}$. Since $\mathrm{g}$ $\neq 0$ and $\mathrm{G}$ is a right R-group of type- $\nu, \mathrm{gR} \neq\{0\}$ as $\{h \in G \mid h R=\{0\}\}$ is an ideal of $G$. Let $<g R>_{s}$ be the subgroup of $(G,+)$ generated by $g R$. Let $\mathrm{h} \in\langle g R\rangle_{s}$. Now $\mathrm{h}=\delta_{1} \mathrm{gs}_{1}+\delta_{2} \mathrm{gs}_{2}+\ldots+\delta_{k} \mathrm{gs}_{k}, \mathrm{~s}_{i} \in \mathrm{R}, \delta_{i} \in\{1$, $-1\} . \mathrm{hr}=\delta_{1} \mathrm{~g}\left(\mathrm{~s}_{1} \mathrm{r}\right)+\delta_{2} \mathrm{~g}\left(\mathrm{~s}_{2} \mathrm{r}\right)+\ldots+\delta_{k} \mathrm{~g}\left(\mathrm{~s}_{k} \mathrm{r}\right) \in<g R>_{s}$. So $<g R>_{s}$ is a non-zero R-subgroup of the right R-group G. Since G is of type- $\nu,\langle g R\rangle_{s}$ $=\mathrm{G}$. Therefore, $\mathrm{hr}_{1}=\mathrm{hr}_{2}$ for all $\mathrm{h} \in \mathrm{G}$ as $\operatorname{gxr}_{1}=\operatorname{gxr}_{2}$ for all $\mathrm{x} \in \mathrm{R}$. So $\mathrm{r}_{1}-$ $\mathrm{r}_{2} \in \mathrm{P}$.

We give an example of a right R-group of type-1(e) which is not of type$2(\mathrm{e})$.

Example 3.5. Let $p$ be an odd prime number and $(G,+)$ be a group of order p. Consider the near-ring $M_{0}(G)$. In Example 3.6 of [8], it is shown that $M_{0}(G)$ is a right $M_{0}(G)$-group of type-1 but not of type-2. Since $M_{0}(G)$ is simple, $\{0\}$ is the largest ideal of $M_{0}(G)$ contained in $\left(0: M_{0}(G)\right)$. Suppose that $0 \neq s, f, h \in M_{0}(G)$ and stf $=$ sth for all $t \in M_{0}(G)$. Assume that $s\left(g_{0}\right) \neq$ 0 and $f(g) \neq h(g)$ for some $g_{0}, g \in G$. Let $h(g) \neq 0$. We get $t \in M_{0}(G)$ such that $t(f(g))=0$ and $t(h(g))=g_{0}$. So stf $\neq$ sth, a contradiction. Therefore, $f$ $=h$, that is, $f-h \in\{0\}$. Hence, $M_{0}(G)$ is a right $M_{0}(G)$-group of type-1(e) but not of type-2(e).

Example 3.6. Clearly, a near-field $R$ is a right $R$-group of type-2(e).

The following Proposition follows from Proposition 3.12 of [13].

Proposition 3.7. Let $G$ be right $R$-group of type- $\nu(e)$. Then $(0: G)$ is an ideal of $R$.

Definition 3.8. A right modular right ideal $\mathrm{K}$ of $\mathrm{R}$ is called right $\nu(e)$-modular if $\mathrm{R} / \mathrm{K}$ is a right $\mathrm{R}$-group of type- $\nu(\mathrm{e})$. 
Definition 3.9. Let $\mathrm{G}$ be a right R-group of type- $\nu(\mathrm{e})$. Then $(0: \mathrm{G})$ is called a right $\nu(e)$-primitive ideal of $\mathrm{R}$.

Definition 3.10. Let $\mathrm{G}$ be a right R-group of type- $\nu(\mathrm{e})$. Then $\mathrm{G}$ is called faithful if $(0: \mathrm{G})=\{0\}$.

Definition 3.11. A near-ring $\mathrm{R}$ is called right $\nu(e)$-primitive if $\{0\}$ is a right $\nu(\mathrm{e})$-primitive ideal of $\mathrm{R}$.

Definition 3.12. The intersection of all $\nu(\mathrm{e})$-primitive ideals of $\mathrm{R}$ is called the right Jacobson radical of $R$ of type- $\nu(e)$ and is denoted by $J_{\nu(e)}^{r}(R)$. If $\mathrm{R}$ has no right $\nu(\mathrm{e})$-primitive ideals, then $J_{\nu(e)}^{r}(\mathrm{R})$ is defined to be $\mathrm{R}$.

Remark 3.13. It is clear that $J_{\nu}^{r}(R) \subseteq J_{\nu(e)}^{r}(R)$.

Proposition 3.14. Let $G$ be a right $R$-group of type- $\nu(e)$. Let $g_{0}$ be a generator of $G$ and $K:=\left(0: g_{0}\right)=\left\{r \in R \mid g_{0} r=0\right\}$. Then $K$ is right $\nu(e)$-modular right ideal of $R$.

Proof. Since $\mathrm{g}_{0} \mathrm{R}=\mathrm{G}, \mathrm{g}_{0}=\mathrm{g}_{0}$ e for some e $\in \mathrm{R}$. So $r-e r \in K$ for all $\mathrm{r} \in \mathrm{R}$ and hence $\mathrm{K}$ is right modular by e. Since the mapping $r \rightarrow g_{0} r$ is right $\mathrm{R}$ homomorphism of $\mathrm{R}$ onto $\mathrm{G}$ with kernel $\mathrm{K}$, the right $\mathrm{R}$-group $\mathrm{G}$ is isomorphic to the right $\mathrm{R}$-group $\mathrm{R} / \mathrm{K}$. So $\mathrm{K}$ is a right $\nu(\mathrm{e})$-modular right ideal of $\mathrm{R}$.

Remark 3.15. Let $\mathrm{K}$ be a right ideal of $\mathrm{R}$. Then the ideal $\{0\}$ of $\mathrm{R}$ is contained in $\mathrm{K}$. Since $\mathrm{K}$ is a subgroup of $(\mathrm{R},+)$ if I and $\mathrm{J}$ are ideals of $\mathrm{R}$ contained in $\mathrm{K}$, then $\mathrm{I}+\mathrm{J} \subseteq \mathrm{K}$. So there is a largest ideal of $\mathrm{R}$ contained in $\mathrm{K}$.

The following Proposition follows from Proposition 3.19 of [13].

Proposition 3.16. Let $G$ be right $R$-group of type- $\nu(e)$ and $P:=(0: G)=$ $\{r \in R \mid G r=\{0\}\}$. Then $P$ is the largest ideal of $R$ contained in $\left(0: g_{0}\right)$, $g_{0}$ is a generator of the right $R$-group $G$.

Corollary 3.17. Let $P$ be an ideal of $R . P$ is a right $\nu(e)$-primitive ideal of $R$ if and only if $P$ is the largest ideal of $R$ contained in a right $\nu(e)$-modular right ideal of $R$.

We give some more examples of right R-groups of type-2(e).

Proposition 3.18. If $G$ be a finite group and $G$ has a subgroup of index two, then $M_{0}(G)$ is a right $2(e)$-primitive near-ring.

Proof. Let $\mathrm{G}$ be a finite group and $\mathrm{H}$ be a subgroup of $\mathrm{G}$ of index 2. So $\mathrm{H}$ is a normal subgroup of $G$. Let $R=M_{0}(G)$. Then $R / K$ is a right $R$-group of type-2(e), where $\mathrm{K}=(\mathrm{H}: \mathrm{G})=\{\mathrm{r} \in \mathrm{R} \mid \mathrm{r}(\mathrm{g}) \in \mathrm{H}$, for all $\mathrm{g} \in \mathrm{G}\}$. To show 
this we consider the two distinct cosets $\mathrm{H}$ and $\mathrm{H}+\mathrm{a}$ of $\mathrm{H}$ in $\mathrm{G}$. Now $\mathrm{G}=\mathrm{H}$ $\cup \mathrm{H}+\mathrm{a}, \mathrm{H}$ and $\mathrm{H}+\mathrm{a}$ are disjoint sets. $\mathrm{K}$ is a right ideal of $\mathrm{R}$ which is right modular by the identity element of $\mathrm{R}$. So $\mathrm{R} / \mathrm{K}$ is a monogenic right $\mathrm{R}$-group. Now we show that $R / K$ is a right $R$-group of type-2. Let $0 \neq r+K \in R / K$. $(\mathrm{r}+\mathrm{K}) \mathrm{R}=\mathrm{R} / \mathrm{K}$ if and only if there is an $\mathrm{s} \in \mathrm{R}$ such that $(\mathrm{r}+\mathrm{K}) \mathrm{s}=1+$ $\mathrm{K}$, that is, $1-\mathrm{rs} \in \mathrm{K}$. Let $\mathrm{P}_{1}=\{\mathrm{x} \in \mathrm{G} \mid \mathrm{r}(\mathrm{x}) \in \mathrm{H}\}$ and $\mathrm{P}_{2}=\{\mathrm{x} \in \mathrm{G} \mid \mathrm{r}(\mathrm{x})$ $\in \mathrm{H}+\mathrm{a}\}$. Let $\mathrm{b} \in \mathrm{P}_{2}$ and $\mathrm{r}(\mathrm{b})=\mathrm{h}^{\prime}+\mathrm{a}, \mathrm{h}^{\prime} \in \mathrm{H}$. Define $\mathrm{s}: \mathrm{G} \rightarrow \mathrm{G}$ by $\mathrm{s}(\mathrm{g})$ $=\mathrm{b}$, if $\mathrm{g} \in \mathrm{H}+\mathrm{a}$, and 0 , if $\mathrm{g} \in \mathrm{H}$. We have $\mathrm{s} \in \mathrm{R}$. For $\mathrm{y} \in \mathrm{H},(1-\mathrm{rs})(\mathrm{y})=$ $\mathrm{y}-\mathrm{r}(\mathrm{s}(\mathrm{y}))=\mathrm{y}-\mathrm{r}(0)=\mathrm{y} \in \mathrm{H}$ and for $\mathrm{z}=\mathrm{h}+\mathrm{a} \in \mathrm{H}+\mathrm{a},(1-\mathrm{rs})(\mathrm{z})=\mathrm{z}-$ $\mathrm{r}(\mathrm{s}(\mathrm{z}))=\mathrm{z}-\mathrm{r}(\mathrm{b})=(\mathrm{h}+\mathrm{a})-\left(\mathrm{h}^{\prime}+\mathrm{a}\right)=\mathrm{h}-\mathrm{h}^{\prime} \in \mathrm{H}$. Therefore, $1-\mathrm{rs} \in(\mathrm{H}:$ $\mathrm{G})=\mathrm{K}$ and hence $\mathrm{R} / \mathrm{K}$ is a right $\mathrm{R}$-group of type- 2 . Since $\mathrm{R}$ is simple, $\{0\}$ is the largest ideal of $\mathrm{R}$ contained in $(0: \mathrm{R} / \mathrm{K})=(\mathrm{K}: \mathrm{R})=\{\mathrm{t} \in \mathrm{R} \mid \mathrm{Rt} \subseteq \mathrm{K}\}$. Let $\mathrm{u}, \mathrm{v} \in \mathrm{R}$ and $(\mathrm{t}+\mathrm{K}) \mathrm{u}=(\mathrm{t}+\mathrm{K}) \mathrm{v}$ for all $\mathrm{t}+\mathrm{K} \in \mathrm{R} / \mathrm{K}$. Now $\mathrm{tu}-\mathrm{tv} \in$ $\mathrm{K}$, for all $\mathrm{t} \in \mathrm{R}$. Suppose that $\mathrm{g} \in \mathrm{G}$ and $\mathrm{u}(\mathrm{g}) \neq \mathrm{v}(\mathrm{g})$. We can choose a $\mathrm{t} \in \mathrm{R}$ such that $(\mathrm{tu})(\mathrm{g})-(\mathrm{tv})(\mathrm{g}) \in \mathrm{H}+\mathrm{a}$, a contradiction to the fact that $\mathrm{tu}-\mathrm{tv} \in$ $\mathrm{K}$. Therefore, $\mathrm{u}=\mathrm{v}$ and hence $\mathrm{R} / \mathrm{K}$ is a right R-group of type-2(e). Since $\mathrm{R}$ is simple, it is a right $2(\mathrm{e})$-primitive near-ring.

Proposition 3.19. If $G$ is a finite group having no subgroup of index 2 , then $J_{2(e)}^{r}\left(M_{0}(G)\right)=M_{0}(G)$.

Proof. Let $\mathrm{G}$ be a finite group having no subgroup of index 2. Let $\mathrm{R}:=$ $\mathrm{M}_{0}(\mathrm{G})$. Suppose that $\mathrm{K}$ is a right 2-modular right ideal of $\mathrm{R}$. Now $\mathrm{K}=(\mathrm{N}$ : $\mathrm{G}$ ), where $\mathrm{N}$ is a normal subgroup of $\mathrm{G}$. By our assumption the index of $\mathrm{N}$ in $\mathrm{G}$ is greater than or equal to 3 . Let $\mathrm{N}, \mathrm{N}+\mathrm{a}, \mathrm{N}+\mathrm{b}$ be three distinct right cosets of $\mathrm{N}$ in $\mathrm{G}$. Since $\mathrm{R} / \mathrm{K}$ is a right R-group of type- 2 , for $0 \neq t+\mathrm{K} \in$ $\mathrm{R} / \mathrm{K},(\mathrm{t}+\mathrm{K}) \mathrm{R}=\mathrm{R} / \mathrm{K}$. Since $1+\mathrm{K} \in \mathrm{R} / \mathrm{K}$, we get $\mathrm{s} \in \mathrm{R}$ such that $(\mathrm{t}+\mathrm{K}) \mathrm{s}$ $=1+\mathrm{K}$, and hence $1-\mathrm{ts} \in \mathrm{K}=(\mathrm{N}: \mathrm{G})$. Define $\mathrm{r}: \mathrm{G} \rightarrow \mathrm{G}$ by $\mathrm{r}(\mathrm{a})=\mathrm{b}$ and $\mathrm{r}(\mathrm{g})=0$ for all $g \in G \backslash\{a\}$. Now $\mathrm{r} \in \mathrm{R}$. If $\mathrm{r} \in \mathrm{K}=(\mathrm{N}: \mathrm{G})$, then $\mathrm{r}(\mathrm{x}) \in \mathrm{N}$ for all $\mathrm{x} \in \mathrm{G}$ and in particular $\mathrm{b}=\mathrm{r}(\mathrm{a}) \in \mathrm{N}$, a contradiction. So $\mathrm{r} \notin \mathrm{K}$ and there is a $p \in R$ such that $1-r p \in K=(N: G)$. Now $(1-r p)(x) \in N$ for all $\mathrm{x} \in \mathrm{G}$. If $\mathrm{p}(\mathrm{a})=\mathrm{a}$, then $(1-\mathrm{rp})(\mathrm{a})=\mathrm{a}-\mathrm{b} \in \mathrm{N}$ and hence $\mathrm{N}+\mathrm{a}=\mathrm{N}+\mathrm{b}, \mathrm{a}$ contradiction. If $\mathrm{p}(\mathrm{a}) \neq \mathrm{a}$, then $(1-\mathrm{rp})(\mathrm{a})=\mathrm{a}-0=\mathrm{a} \in \mathrm{N}$ and $\mathrm{N}=\mathrm{N}+\mathrm{a}$, a contradiction. Therefore, $\mathrm{R}$ has no right 2 -modular right ideal. So, $\mathrm{J}_{2}^{r}(\mathrm{R})=$ $\mathrm{R}$ and hence $\mathrm{J}_{2(e)}^{r}(\mathrm{R})=\mathrm{R}$.

Proposition 3.20. If $F$ is a near-field, then $M_{n}(F)$ is a right 2(e)-primitive near-ring.

Proof. Let $\mathrm{F}$ be a near-field. Let $\mathrm{M}_{n}(\mathrm{~F})$ be the near-ring of $\mathrm{n} \times \mathrm{n}$-matrices over F. Let $1 \leq \mathrm{i} \leq \mathrm{n}$. Now from the proof of the Theorem 3.15 of [6], we have that $\mathrm{f}_{i i}^{1} \mathrm{M}_{n}(\mathrm{~F})$ is a right $\mathrm{M}_{n}(\mathrm{~F})$-group of type-2. Since $\mathrm{M}_{n}(\mathrm{~F})$ is simple, $\{0\}$ is the largest ideal of $\mathrm{M}_{n}(\mathrm{~F})$ contained in $\left(0: \mathrm{f}_{i i}^{1} \mathrm{M}_{n}(\mathrm{~F})\right)$. We show now that 
$\mathrm{f}_{i i}^{1} \mathrm{M}_{n}(\mathrm{~F})$ is a right $\mathrm{M}_{n}(\mathrm{~F})$-group of type-2(e). Let $\mathrm{B}, \mathrm{C} \in \mathrm{M}_{n}(\mathrm{~F})$ and $\left(\mathrm{f}_{i i}^{1} \mathrm{~A}\right) \mathrm{B}$ $=\left(\mathrm{f}_{i i}^{1} \mathrm{~A}\right) \mathrm{C}$, for all $\mathrm{A} \in \mathrm{M}_{n}(\mathrm{~F})$. Suppose that $\mathrm{B} \neq \mathrm{C}$. We get $\left(\mathrm{x}_{1}, \mathrm{x}_{2}, \ldots, \mathrm{x}_{n}\right)$ $\in \mathrm{F}^{n}$ such that $\mathrm{B}\left(\mathrm{x}_{1}, \mathrm{x}_{2}, \ldots, \mathrm{x}_{n}\right) \neq \mathrm{C}\left(\mathrm{x}_{1}, \mathrm{x}_{2}, \ldots, \mathrm{x}_{n}\right)$. Let $\mathrm{B}\left(\mathrm{x}_{1}, \mathrm{x}_{2}, \ldots, \mathrm{x}_{n}\right)$ $=\left(\mathrm{y}_{1}, \mathrm{y}_{2}, \ldots, \mathrm{y}_{n}\right)$ and $\mathrm{C}\left(\mathrm{x}_{1}, \mathrm{x}_{2}, \ldots, \mathrm{x}_{n}\right)=\left(\mathrm{z}_{1}, \mathrm{z}_{2}, \ldots, \mathrm{z}_{n}\right)$. We get $1 \leq \mathrm{j} \leq$ $\mathrm{n}$ such that $\mathrm{y}_{j} \neq \mathrm{z}_{j}$. Now $\left(\mathrm{f}_{i i}^{1} \mathrm{f}_{i j}^{1}\right) \mathrm{B}\left(\mathrm{x}_{1}, \mathrm{x}_{2}, \ldots, \mathrm{x}_{n}\right)=\left(\mathrm{f}_{i i}^{1} \mathrm{f}_{i j}^{1}\right) \mathrm{C}\left(\mathrm{x}_{1}, \mathrm{x}_{2}, \ldots, \mathrm{x}_{n}\right)$ and that $\mathrm{y}_{j}=\mathrm{z}_{j}$, a contradiction. Therefore $\mathrm{B}=\mathrm{C}$ and hence $\mathrm{f}_{i i}^{1} \mathrm{M}_{n}(\mathrm{~F})$ is a right $\mathrm{M}_{n}(\mathrm{~F})$-group of type- $2(\mathrm{e})$. Since $\mathrm{F}$ is simple, $\mathrm{M}_{n}(\mathrm{~F})$ is also simple. So, we get that $\mathrm{M}_{n}(\mathrm{~F})$ is a right 2(e)-primitive near-ring.

Now we give a right R-group of type-2(e), where $\mathrm{R}$ is a near-ring with trivial multiplication.

Example 3.21. Let $(R,+)$ be a group and let $K$ be a subgroup of $(R,+)$ of index 2. The trivial multiplication on $(R,+)$ determined by $R-K$ is given by $a . b=a$ if $b \in R-K$ and 0 if $b \in K$. Now $(R,+,$.$) is a near-ring. It is$ clear that $K$ is a maximal right ideal of $R$ and also $R / K$ is a right $R$-group of type-2. Now we show that $R / K$ is a right $R$-group of type-2(e). $K$ is an ideal of $R$ and it is the largest ideal of $R$ contained in $K$ and hence in $(K: R)=$ $\{r \in R \mid R r \subseteq K\}$. Let $x, y \in R$ and $(r+K) x=(r+K) y$ for all $r \in R$. Now $r x-r y \in K$ for all $r \in R$. So, either both $x$ and $y$ are in $K$ or both in $R$ $K$. Therefore, $x-y \in K$ as $K$ is of index 2 in $(R,+)$. Hence, $R / K$ is a right $R$-group of type-2(e).

Now we give an example of a right R-group of type- $\nu$ which is not of type$\nu(\mathrm{e})$.

This example was considered in [3] and [13].

Example 3.22. Consider $G:=Z_{8}$, the group of integers under addition modulo 8. Now $T: G \rightarrow G$ defined by $T(g)=5 g$, for all $g \in G$ is an automorphism of $G$. T fixes 0, 2, 4, 6 and maps 1 to 5, 5 to 1,7 to 3 and 3 to $7 . A:=\{I$, $T\}$ is an automorphism group of $G .\{0\},\{2\},\{4\},\{6\},\{1,5\}$ and $\{3,7\}$ are the orbits. Let $R$ be the centralizer near-ring $M_{A}(G)$, the near-ring of all self maps of $G$ which fix 0 and commute with $T$. An element of $R$ is completely determined by its action on $\{1,2,3,4,6\}$. Note that for $f \in R$ we have $f(2)$, $f(4), f(6)$ are arbitrary in $2 G$ and $f(1), f(3)$ are arbitrary in $G$. In [3] it is proved that $I:=(0: 2 G)=\{f \in R \mid f(h)=0$, for all $h \in 2 G\}$ is the only non-trivial ideal of $R$. Let $K:=(2 G: G)=\{t \in R \mid t(G) \subseteq 2 G\} \neq R$. Let $t_{0}$ be the identity element in $R$. Now $t_{0}+K$ is a generator of the right $R$-group $R / K$. Let $h \in R-K$. We show now that $(h+K) R=R / K$. Since $h \notin K$, there is an $a \in G-2 G$ such that $b:=h(a) \notin 2 G$. We construct an element $s \in R$ such that $s(1)=s(3)=a$, so that $s(5)=s(7)=a+4$, and $s=0$ on $2 G$. Since s maps $G-2 G$ to $G-2 G$, we get that $t_{0}-h s \in K$ and hence $(h+K) s$ $=t_{0}+K$. So $(h+K) R=R / K$. Therefore, $R / K$ is a right $R$-group of type- $\nu$. 
Moreover, $(R / K) I \neq\{K\}$. Therefore, $\{0\}$ is the largest ideal of $R$ contained in $(K: R)$ and hence $J_{\nu}^{r}(R)=\{0\}$. Consider $s_{1}, s_{1} \in R$, where $s_{1}(1)=1$ and 0 on $G-\{1,5\}$ and $s_{2}(1)=5$ and 0 on $G-\{1,5\}$. Clearly $(h+K) s_{1}=(h$ $+K) s_{2}$ for all $h \in R$ as $h(1)-h(5) \in 2 G$ for all $h \in R$. But $s_{1}-s_{2} \notin\{0\}$. Therefore, by Proposition 3.4, $R / K$ is not a right $R$-group of type- $\nu(e)$.

Proposition 3.23. Let $R$ be the near-ring considered in the Example 3.22 and let $Z$ be a right ideal of $R$. Then $H_{1}:=\{f(g) \mid f \in Z, g \in G\} \subseteq G$ and $H_{2}:=\{f(g) \mid f \in Z, g \in 2 G\} \subseteq 2 G$ are (normal) subgroups of $G$ and $2 G$ respectively.

Proof. We show that $\mathrm{H}_{1}$ is a subgroup of $\mathrm{G}$. Since $0 \in \mathrm{H}_{1}, \mathrm{H}_{1}$ is non-empty. Let $h_{1}, h_{2} \in H_{1}$. We get $f_{1}, f_{2} \in Z$ and $g_{1}, g_{2} \in G$ such that $h_{1}=f_{1}\left(g_{1}\right)$ and $\mathrm{h}_{2}=\mathrm{f}_{2}\left(\mathrm{~g}_{2}\right)$. Clearly, $-\mathrm{h}_{1}=\left(-\mathrm{f}_{1}\right)\left(\mathrm{g}_{1}\right) \in \mathrm{H}_{1}$ as $-\mathrm{f}_{1} \in \mathrm{Z}$. Suppose that one of the $\mathrm{g}_{i}$ is in $\mathrm{G}-2 \mathrm{G}$. With out loss of generality, suppose that $\mathrm{g}_{1} \in \mathrm{G}-2 \mathrm{G}$. We get $\mathrm{f}_{3} \in \mathrm{R}$ such that $\mathrm{f}_{3}\left(\mathrm{~g}_{1}\right)=\mathrm{g}_{2}$. Now $\mathrm{f}_{1}-\mathrm{f}_{2} \mathrm{f}_{3} \in \mathrm{Z}$ and $\mathrm{h}_{1}-\mathrm{h}_{2}=\left(\mathrm{f}_{1}-\mathrm{f}_{2} \mathrm{f}_{3}\right)\left(\mathrm{g}_{1}\right) \in$ $\mathrm{H}_{1}$. Assume now that $\mathrm{g}_{1}, \mathrm{~g}_{2} \in 2 \mathrm{G}$. So, $\mathrm{h}_{1}, \mathrm{~h}_{2} \in 2 \mathrm{G}$. If $\mathrm{g}_{1}=0$, then $\mathrm{h}_{1}-\mathrm{h}_{2}=$ $-\mathrm{h}_{2} \in \mathrm{H}_{1}$. Suppose that $\mathrm{g}_{1} \neq 0$. So, we get $\mathrm{f}_{4} \in \mathrm{R}$ such that $\mathrm{f}_{4}\left(\mathrm{~g}_{1}\right)=\mathrm{g}_{2}$. Now $\mathrm{f}_{1}-\mathrm{f}_{2} \mathrm{f}_{4} \in \mathrm{Z}$ and $\mathrm{h}_{1}-\mathrm{h}_{2}=\left(\mathrm{f}_{1}-\mathrm{f}_{2} \mathrm{f}_{4}\right)\left(\mathrm{g}_{1}\right) \in \mathrm{H}_{1}$. Therefore, $\mathrm{H}_{1}$ is a subgroup of G. Similarly, we get that $\mathrm{H}_{2}$ is a subgroup of $2 \mathrm{G}$.

Proposition 3.24. Let $R, Z, H_{1}$ and $H_{2}$ be as defined in Proposition 3.23. If $H_{1}=G$ and $H_{2}=2 G$, then $Z=R$.

Proof. Suppose that $\mathrm{H}_{1}=\mathrm{G}$ and $\mathrm{H}_{2}=2 \mathrm{G}$. We have $1,3 \in \mathrm{H}_{1}$. So, for $\mathrm{i} \in$ $\{1,3\}$, we get $\mathrm{f}_{i} \in \mathrm{Z}$ such that $\mathrm{f}_{i}\left(\mathrm{~g}_{i}\right)=\mathrm{i}$, where $\mathrm{g}_{i} \in\{1,3,5,7\}=\mathrm{G}-2 \mathrm{G}$. For $\mathrm{i}=1,3$ we also get $\mathrm{m}_{i} \in \mathrm{R}$ such that $\mathrm{m}_{i}(\mathrm{i})=\mathrm{g}_{i}$, so that $\mathrm{m}_{i}(\mathrm{i}+4)=$ $\mathrm{g}_{i}+4$ and $\mathrm{m}_{i}=0$ on $\mathrm{G}-\{\mathrm{i}, \mathrm{i}+4\}$. Now $\mathrm{f}_{i} \mathrm{~m}_{i} \in \mathrm{Z}, \mathrm{i}=1,3$. Clearly, $\mathrm{f}_{1} \mathrm{~m}_{1}$ $+\mathrm{f}_{3} \mathrm{~m}_{3}$ fixes all the elements of $\mathrm{G}-2 \mathrm{G}$ and maps all the elements of $2 \mathrm{G}$ to 0 . We have $2,4,6 \in \mathrm{H}_{2}=2 \mathrm{G}=\{0,2,4,6\}$. For $\mathrm{i}=2,4,6$ we get $\mathrm{f}_{i} \in \mathrm{Z}$ such that $\mathrm{f}_{i}\left(\mathrm{~g}_{i}\right)=\mathrm{i}, \mathrm{g}_{i} \in 2 \mathrm{G}$. So, for $\mathrm{i}=2,4,6$ we get $\mathrm{m}_{i} \in \mathrm{R}$ such that $\mathrm{m}_{i}(\mathrm{i})=\mathrm{g}_{i}$ and $\mathrm{m}_{i}$ is 0 on $\mathrm{G}-\{\mathrm{i}\}$. Now $\mathrm{f}_{i} \mathrm{~m}_{i} \in \mathrm{Z}, \mathrm{i}=2,4,6 . \mathrm{f}_{2} \mathrm{~m}_{2}+\mathrm{f}_{4} \mathrm{~m}_{4}+\mathrm{f}_{6} \mathrm{~m}_{6}$ fixes all the elements of $2 \mathrm{G}$ and maps all the elements of $\mathrm{G}-2 \mathrm{G}$ to 0 . Therefore, the identity map $I$ of $G$ can be expressed as $I=f_{1} m_{1}+f_{2} m_{2}+f_{3} m_{3}+f_{4} m_{4}$ $+\mathrm{f}_{6} \mathrm{~m}_{6} \in \mathrm{Z}$. Hence, $\mathrm{Z}=\mathrm{R}$.

Proposition 3.25. Let $R, Z, H_{1}$ and $H_{2}$ be as defined in Proposition 3.23. If $Z$ is a maximal right ideal of $R$, then $Z=(2 G: G)=\{f \in R \mid f(G) \subseteq 2 G\}$ or $(4 G: 2 G)=\{f \in R \mid f(2 G) \subseteq 4 G\}$

Proof. Suppose that $\mathrm{Z}$ is a maximal right ideal of $\mathrm{R}$. Clearly, if $\mathrm{H}$ and $\mathrm{T}$ are (normal) subgroups of $G$ and $2 G$ respectively, then $(H: G)=\{f \in R \mid f(G)$ $\subseteq \mathrm{H}\}$ and $(\mathrm{T}: 2 \mathrm{G})=\{\mathrm{f} \in \mathrm{R} \mid \mathrm{f}(2 \mathrm{G}) \subseteq \mathrm{T}\}$ are right ideals of $\mathrm{R}$. Now $2 \mathrm{G}$ and $4 \mathrm{G}$ are the maximal (normal) subgroups of $\mathrm{G}$ and $2 \mathrm{G}$ respectively. We have 
$\mathrm{Z} \subseteq\left(\mathrm{H}_{1}: \mathrm{G}\right)$ and $\mathrm{Z} \subseteq\left(\mathrm{H}_{2}: 2 \mathrm{G}\right)$. Since $\mathrm{Z}$ is a maximal right ideal of $\mathrm{R}$, by Proposition 3.24, either $\mathrm{H}_{1} \neq \mathrm{G}$ or $\mathrm{H}_{2} \neq 2 \mathrm{G}$.

Case(i) Suppose that $\mathrm{H}_{2} \neq 2 \mathrm{G}$. Since $\mathrm{Z}$ is a maximal right ideal of $\mathrm{R}$ and $\mathrm{Z}$ $\subseteq\left(\mathrm{H}_{2}: 2 \mathrm{G}\right) \neq \mathrm{R}$, we get that $\mathrm{H}_{2}=4 \mathrm{G}$ and $\mathrm{Z}=(4 \mathrm{G}: 2 \mathrm{G})$.

case(ii) Suppose that $\mathrm{H}_{1} \neq \mathrm{G}$. Since $\mathrm{Z}$ is a maximal right ideal of $\mathrm{R}$ and $\mathrm{Z} \subseteq$ $\left(\mathrm{H}_{1}: \mathrm{G}\right) \neq \mathrm{R}$, we get that $\mathrm{H}_{1}=2 \mathrm{G}$ and $\mathrm{Z}=(2 \mathrm{G}: \mathrm{G})$.

Therefore, either $\mathrm{Z}=(2 \mathrm{G}: \mathrm{G})$ or $(4 \mathrm{G}: 2 \mathrm{G})$.

Proposition 3.26. Let $R$ be the near-ring considered in the Example 3.22. Let $U=(4 G: 2 G)=\{f \in R \mid f(2 G) \subseteq 4 G\}$. Then $U$ is a maximal right ideal of $R$ and $R / U$ is a right $R$-group of type-2(e).

Proof. Clearly, $\mathrm{U}$ is a right ideal of $\mathrm{R}$. Consider the right $\mathrm{R}$-group $\mathrm{R} / \mathrm{U}$. We prove that $R / U$ is a right $R$-group of type- 2 . Since $R$ has identity $I, I+U$ is a generator of the right $R$-group $R / U$ and hence $R / U$ is a monogenic right R-group. Let $0 \neq \mathrm{f}+\mathrm{U} \in \mathrm{R} / \mathrm{U}$. So, $\mathrm{f} \notin \mathrm{U}$. We get $0 \neq \mathrm{a} \in 2 \mathrm{G}$ such that $\mathrm{b}:=$ $\mathrm{f}(\mathrm{a}) \notin 4 \mathrm{G}$. So, $2 \mathrm{G}=\{0, \mathrm{~b}, 2 \mathrm{~b}, 3 \mathrm{~b}\}$ as 2 and 6 are generators of $2 \mathrm{G}$. Construct $\mathrm{r} \in \mathrm{R}$ by $\mathrm{r}(\mathrm{b})=\mathrm{a}, \mathrm{r}(2 \mathrm{~b})=0, \mathrm{r}(3 \mathrm{~b})=\mathrm{a}$ and $\mathrm{r}=0$ on $\mathrm{G}-\{0,1,3,5,7\}$. Now $(\mathrm{I}-\mathrm{fr})(\mathrm{x}) \in 4 \mathrm{G}$ for all $\mathrm{x} \in 2 \mathrm{G}$. Therefore, $\mathrm{I}-\mathrm{fr} \in \mathrm{U}$ and hence $(\mathrm{f}+\mathrm{U}) \mathrm{r}=\mathrm{I}$ $+\mathrm{U}$. This shows that $(\mathrm{f}+\mathrm{U}) \mathrm{R}=\mathrm{R} / \mathrm{U}$. So, $\mathrm{R} / \mathrm{U}$ is a right $\mathrm{R}$-group of type-2. We know that $\mathrm{P}:=(0: 2 \mathrm{G})$ is the only non-trivial ideal of $\mathrm{R}$. Therefore, $\mathrm{P}$ is the largest ideal of $\mathrm{R}$ contained in $\mathrm{U}=(4 \mathrm{G}: 2 \mathrm{G})$ and hence $\mathrm{P}$ is the largest ideal of $\mathrm{R}$ contained in $(0: \mathrm{R} / \mathrm{U})=(\mathrm{U}: \mathrm{R})=\{\mathrm{f} \in \mathrm{R} \mid \mathrm{Rf} \subseteq \mathrm{U}\}$. Let $0 \neq \mathrm{s}+$ $\mathrm{U} \in \mathrm{R} / \mathrm{U}$ and $\mathrm{f}, \mathrm{h} \in \mathrm{R}$. Suppose that $(\mathrm{s}+\mathrm{U}) \mathrm{rf}=(\mathrm{s}+\mathrm{U}) \mathrm{rh}$ for all $\mathrm{r} \in \mathrm{R}$. So, srf - srh $\in \mathrm{U}$ for all $\mathrm{r} \in \mathrm{R}$. We show that $\mathrm{f}-\mathrm{h} \in \mathrm{P}$. If possible, suppose that $\mathrm{f}$ - $\mathrm{h} \notin \mathrm{P}$. We get $0 \neq \mathrm{a} \in 2 \mathrm{G}$ such that $(\mathrm{f}-\mathrm{h})(\mathrm{a})=\mathrm{f}(\mathrm{a})-\mathrm{h}(\mathrm{a}) \neq 0$ with $\mathrm{h}(\mathrm{a}) \neq$ 0 . Let $\mathrm{s}(\mathrm{c}) \notin\{0,4\}$ for some $\mathrm{c} \in 2 \mathrm{G}$. Choose $\mathrm{r} \in \mathrm{R}$ such that $\mathrm{r}(\mathrm{f}(\mathrm{a}))=0$ and $\mathrm{r}(\mathrm{h}(\mathrm{a}))=\mathrm{c}$. Now $(\operatorname{srf})(\mathrm{a})=0$ and $(\operatorname{srh})(\mathrm{a})=\mathrm{s}(\mathrm{c})$. So, $(\operatorname{srf}-\mathrm{srh})(\mathrm{a})=0-\mathrm{s}(\mathrm{c})$ $\notin\{0,4\}$, a contradiction to the fact that srf - srh $\in \mathrm{U}$. Therefore, $\mathrm{f}(\mathrm{a})=\mathrm{h}(\mathrm{a})$ for all $\mathrm{a} \in 2 \mathrm{G}$. Hence $\mathrm{f}-\mathrm{h} \in \mathrm{P}$. So, $\mathrm{R} / \mathrm{U}$ is a right $\mathrm{R}$-group of type-2(e).

Proposition 3.27. Let $R$ be the near-ring considered in Example 3.22. Then $J_{\nu}^{r}(R)=\{0\}$ and $J_{\nu(e)}^{r}(R)=(0: 2 G) \neq\{0\}$.

Proof. We know that $\{0\}$ and $\mathrm{I}:=(0: 2 \mathrm{G})=\{\mathrm{f} \in \mathrm{R} \mid \mathrm{f}(2 \mathrm{G})=\{0\}\}$ are the only proper ideals of $R$. Let $K_{1}:=(2 G: G)=\{f \in R \mid f(G) \subseteq 2 G\}$ and $K_{2}$ $:=(4 \mathrm{G}: 2 \mathrm{G})=\{\mathrm{f} \in \mathrm{R} \mid \mathrm{f}(2 \mathrm{G}) \subseteq 4 \mathrm{G}\}$. By Proposition 3.25, a maximal right ideal of $R$ is either $K_{1}$ or $K_{2}$. So, a right $R$-group of type- 0 is isomorphic to $\mathrm{R} / \mathrm{K}_{1}$ or $\mathrm{R} / \mathrm{K}_{2}$. By Example $3.22, \mathrm{R} / \mathrm{K}_{1}$ is a right $\mathrm{R}$-group of type- 2 but not of type-2(e). Since $\{0\}$ is the largest ideal of $R$ contained in $K_{1},\{0\}$ is a right 2-primitive ideal of $\mathrm{R}$ but not a right $2(\mathrm{e})$-primitive ideal of $\mathrm{R}$. By Proposition $3.26, R / K_{2}$ is a right $R$-group of type- $2(e)$. Since $I=(0: 2 G)$ is 
the largest ideal of $\mathrm{R}$ contained in $\mathrm{K}_{2}$, I is a right 2(e)-primitive ideal of $\mathrm{R}$. Therefore, $\mathrm{J}_{\nu}^{r}(\mathrm{R})=\{0\}$ and $\mathrm{J}_{\nu(e)}^{r}(\mathrm{R})=(0: 2 \mathrm{G})$.

Now we study some of the properties of the radical $\mathrm{J}_{\nu(e)}^{r}$.

Proposition 3.28. Let $P$ be an ideal of $R$. $P$ is a right $\nu(e)$-primitive ideal of $R$ if and only if $R / P$ is a right $\nu(e)$-primitive near-ring.

A proof similar to the one given for Proposition 3.21 of [13] works here also, which uses Corollary 3.17.

Theorem 3.29. Let $R$ be a right $\nu(e)$-primitive near-ring. Then $R$ is an equiprime near-ring.

Proof. Since $\{0\}$ is a right $\nu(\mathrm{e})$-primitive ideal of $\mathrm{R}$, by Proposition $3.7,\{0\}$ $=(0: \mathrm{G})$ for a right R-group $\mathrm{G}$ of type- $\nu(\mathrm{e})$. Let $a \in R \backslash\{0\}, \mathrm{r}_{1}, \mathrm{r}_{2} \in \mathrm{R}$ and $\operatorname{axr}_{1}=\operatorname{axr}_{2}$ for all $\mathrm{x} \in \mathrm{R}$. Since $(0: \mathrm{G})=\{0\}$, there is a $\mathrm{g} \in \mathrm{G}$ such that ga $\neq 0$. Let $\mathrm{h}:=$ ga. Now $\mathrm{hxr}_{1}=\mathrm{hxr}_{2}$ for all $\mathrm{x} \in \mathrm{R}$. Since $\mathrm{G}$ is a right $\mathrm{R}$-group of type- $\nu(\mathrm{e}), \mathrm{r}_{1}-\mathrm{r}_{2} \in \mathrm{P}$, the largest ideal of $R$ contained in $(0: \mathrm{G})=\{0\}$. Therefore, $r_{1}=r_{2}$ and hence $R$ is an equiprime near-ring.

Corollary 3.30. A right $\nu(e)$-primitive ideal of $R$ is an equiprime ideal of $R$.

Corollary 3.31. A right $\nu(e)$-primitive near-ring is a zero-symmetric nearring.

Theorem 3.32. Let $G$ be a right $R$-group of type- $\nu(e)$. Suppose that $S$ is an invariant subnear-ring of $R$. If $G S \neq\{0\}$, then $G$ is also a right $S$-group of type- $\nu(e)$.

Proof. Suppose that GS $\neq\{0\}$. By Theorem 2.5, G is a right S-group of type- $\nu$. Let $\mathrm{P}$ be the largest ideal of $\mathrm{S}$ contained in $(0: \mathrm{G})_{S}=\{\mathrm{s} \in \mathrm{S} \mid \mathrm{Gs}=$ $\{0\}\}$. Let $g \in G \backslash\{0\}, \mathrm{s}_{1}, \mathrm{~s}_{2} \in \mathrm{S}$ and $\operatorname{gxs}_{1}=\operatorname{gxs}_{2}$ for all $\mathrm{x} \in \mathrm{S}$. Let $\mathrm{r} \in \mathrm{R}$. Fix $\mathrm{x} \in \mathrm{S}$. We have $\mathrm{g}(\mathrm{rx}) \mathrm{s}_{1}=\mathrm{g}(\mathrm{rx}) \mathrm{s}_{2}$. So $\operatorname{gr}\left(\mathrm{xs}_{1}\right)=\operatorname{gr}\left(\mathrm{xs}_{2}\right)$. Since $\mathrm{G}$ is a right R-group of type- $\nu(\mathrm{e})$, by Proposition 3.7, $\mathrm{xs}_{1}-\mathrm{xs}_{2} \in(0: \mathrm{G})=\{\mathrm{r} \in \mathrm{R} \mid \mathrm{Gr}$ $=\{0\}\}$ which is an ideal of $\mathrm{R}$. Let $\mathrm{g}_{0}$ be a generator of the right S-group $\mathrm{G}$. Now $\mathrm{g}_{0}\left(\mathrm{xs}_{1}-\mathrm{xs}_{2}\right)=0$ and hence $\mathrm{g}_{0} \mathrm{xs}_{1}=\mathrm{g}_{0} \mathrm{xs}_{2}$. Since $\mathrm{g}_{0} \mathrm{~S}=\mathrm{G}$, we have $\mathrm{g}_{0} \mathrm{R}$ $=\mathrm{G}$. So $\mathrm{g}_{0} \mathrm{rs}_{1}=\mathrm{g}_{0} \mathrm{rs}_{2}$, for all $\mathrm{r} \in \mathrm{R}$. Since $\mathrm{G}$ is a right R-group of type- $\nu(\mathrm{e})$, by Proposition 3.7, $\mathrm{s}_{1}-\mathrm{s}_{2} \in(0: \mathrm{G})$. We have $(0: \mathrm{G})_{S}=(0: \mathrm{G}) \cap \mathrm{S}$ is an ideal of $\mathrm{S}$ and hence $\mathrm{P}=(0: \mathrm{G})_{S}$. Now $\mathrm{s}_{1}-\mathrm{s}_{2} \in(0: \mathrm{G}) \cap \mathrm{S}=\mathrm{P}$. Therefore, $\mathrm{G}$ is a right $\mathrm{S}$-group of type- $\nu(\mathrm{e})$.

Theorem 3.33. If $R$ is a right $\nu(e)$-primitive near-ring and $I$ is a nonzero ideal (or a nonzero invariant subnear-ring) of $R$, then $I$ is a right $\nu(e)$-primitive near-ring. 
Theorem 3.34. The class of all right $\nu(e)$-primitive near-rings is hereditary.

Corollary 3.35. The class of all right $\nu(e)$-primitive near-rings is regular.

Theorem 3.36. Let $I$ be an essential left invariant ideal of $R$. If I is a right $\nu(e)$-primitive near-ring, then $R$ is also a right $\nu(e)$-primitive near-ring.

Proof. Suppose that I is a right $\nu(\mathrm{e})$-primitive near-ring and $\mathrm{G}$ is a faithful right I-group of type- $\nu(\mathrm{e})$. Let $\mathrm{r}, \mathrm{s} \in \mathrm{R}$. Let $\mathrm{g}_{0}$ be a generator of the right I-group G. Define gr $:=\mathrm{g}_{0}(\mathrm{ar})$, if $\mathrm{g}=\mathrm{g}_{0} \mathrm{a}, \mathrm{a} \in \mathrm{I}$. By Theorem 2.6, $\mathrm{G}$ is a right R-group of type- $\nu$. Suppose that $g \in G \backslash\{0\}, \mathrm{r}, \mathrm{s} \in \mathrm{R}$ and gxr $=$ gxs, for all $\mathrm{x} \in \mathrm{R}$. Fix $\mathrm{a} \in \mathrm{I}$. Now $\mathrm{g}((\mathrm{ba}) \mathrm{r})=\mathrm{g}((\mathrm{ba}) \mathrm{s})$ and hence $\mathrm{g}(\mathrm{b}(\mathrm{ar}))=\mathrm{g}(\mathrm{b}(\mathrm{as}))$ for all $\mathrm{b} \in \mathrm{I}$. Since $\mathrm{G}$ is a faithful right I-group of type- $\nu(\mathrm{e})$, ar - as $=0$, that is, ar $=$ as. Now ar $=$ as for all $\mathrm{a} \in \mathrm{I}$. Since I is a right $\nu(\mathrm{e})$-primitive near-ring, by Theorem 3.33, I is an equiprime near-ring. Also, since I is an essential left invariant ideal of $\mathrm{R}$, by Proposition 2.3, we get that $\mathrm{R}$ is an equiprime nearring. Since $R$ is equiprime and ar $=$ as for all $a \in I$ and $I$ is a left invariant ideal of $R$, we get that $r=s$. So, $0=r-s \in P$, where $P$ is the largest ideal of $\mathrm{R}$ contained in $(0: \mathrm{G})=\{\mathrm{r} \in \mathrm{R} \mid \mathrm{Gr}=\{0\}\}$. Therefore $\mathrm{G}$ is a right $\mathrm{R}$-group of type- $\nu(\mathrm{e})$. Let $\mathrm{t} \in(0: \mathrm{G})$. Now $\mathrm{Gt}=0$. So $\mathrm{g}_{0}(\mathrm{at})=0$, for all $\mathrm{a} \in \mathrm{I}$ and hence $0=\mathrm{g}_{0}((\mathrm{ba}) \mathrm{t})=\mathrm{g}_{0}(\mathrm{~b}(\mathrm{at}))=\left(\mathrm{g}_{0} \mathrm{~b}\right)$ at for all $\mathrm{a}, \mathrm{b} \in \mathrm{I}$. Since $\mathrm{g}_{0} \mathrm{I}=\mathrm{G}$, we have $\mathrm{G}(\mathrm{at})=0$ for all $\mathrm{a} \in \mathrm{I}$ and hence $\mathrm{It}=0$, as $(0: \mathrm{G})_{I}=0$. Also, since at $=0=\mathrm{a} 0$ for all $\mathrm{a} \in \mathrm{I}$ and $\mathrm{I}$ is an invariant subnear-ring of $\mathrm{R}$ and $\mathrm{R}$ is an equiprime near-ring, we get that $t=0$. Therefore, $\mathrm{G}$ is a faithful right $\mathrm{R}$-group of type- $\nu(\mathrm{e})$ and hence $\mathrm{R}$ is a right $\nu(\mathrm{e})$-primitive near-ring.

Theorem 3.37. The class of all right $\nu(e)$-primitive near-rings is closed under essential left invariant extensions.

Remark 3.38. By Proposition 2.4, the class of all equiprime near-rings satisfy condition $\mathrm{F}_{l}$. So, the class of all $\nu(\mathrm{e})$-primitive near-rings which is also a class of all equiprime near-rings also satisfy condition $\mathrm{F}_{l}$.

By Theorem 2.1, Corollaries 3.31, and 3.35, Theorem 3.37 and Remark 3.38, we get the following:

Theorem 3.39. Let $\mathcal{E}$ be the class of all right $\nu(e)$-primitive near-rings and $\mathcal{U} \mathcal{E}$ be the upper radical class determined by $\mathcal{E}$. Then $\mathcal{U} \mathcal{E}$ is a c-hereditary Kurosh-Amitsur radical class in the variety of all near-rings with hereditary semisimple class $\mathcal{S} \mathcal{U} \mathcal{E}=\overline{\mathcal{E}}$. So, $J_{\nu(e)}^{r}$ is a Kurosh-Amitsur radical in the class of all near-rings and for any ideal I of $R, J_{\nu(e)}^{r}(I) \subseteq J_{\nu(e)}^{r}(R) \cap I$ with equality if I is left invariant.

Corollary 3.40. $J_{\nu(e)}^{r}$ is an ideal-hereditary Kurosh-Amitsur radical in the class of all zero-symmetric near-rings.

Corollary 3.41. $J_{\nu(e)}^{r}$ is a special radical in the class of all near-rings. 


\section{Acknowledgment}

The first author would like to thank the Management of the Nagarjuna Education Society, Guntur, for providing necessary facilities. The first author also acknowledge U.G.C., New Delhi, for the Major Research Project Grant No. F. No. 39-51/2010 (SR), dated 24/12/2010.

\section{References}

[1] Booth, G.L., Groenewald, N.J. and Veldsman, S., A Kurosh-Amitsur prime radical for near-rings, Comm. in Algebra 18 (1990), 3111-3122.

[2] Booth, G.L. and Groenewald, N.J., Special radicals of near-rings, Math. Japonica 37 (1992), no. 4, 702-706.

[3] Kaarli, K., On Jacobson type radicals of near-rings, Acta Math. Hung. 50 (1987), 71-78.

[4] Pilz, G., Near-rings, revised edition, North-Holland, Amsterdam, 1983.

[5] Srinivasa Rao, R., On near-rings with matrix units, Quaest. Math. 17 (1994), no. 3, 321-332.

[6] Srinivasa Rao, R., Wedderburn-Artin theorem analogue for near-rings, Southeast Asian Bull. Math. 27 (2004), no. 5, 915-922.

[7] Srinivasa Rao, R. and Siva Prasad, K., A radical for right near-rings: The right Jacobson radical of type-0, Int. J. Math. Math. Sci. 2006 (2006), no. 16, Article ID 68595, Pages 1-13.

[8] Srinivasa Rao, R. and Siva Prasad, K., Two more radicals for right nearrings: The right Jacobson radicals of type-1 and 2, Kyungpook Math. J. 46 (2006), no. 4, 603-613.

[9] Srinivasa Rao, R. and Siva Prasad, K., A radical for right near-rings: The right Jacobson radical of type-s, Southeast Asian Bull. Math. 32 (2008), no. 3, 509-519.

[10] Srinivasa Rao, R. and Siva Prasad, K., Kurosh-Amitsur right Jacobson radical of type-0 for right near-rings, Int. J. Math. Math. Sci. 2008 (2008), Article ID 741609, Pages 1-6.

[11] Srinivasa Rao, R., Siva Prasad, K. and Sinivas, T., Kurosh-Amitsur right Jacobson radicals of type-1 and 2 for right near-rings, Result. Math. 51 (2008), no. 3-4, 309-317. 
[12] Srinivasa Rao, R. and Siva Prasad, K., Right semisimple right near-rings, Southeast Asian Bull. Math. 33 (2009), no. 6, 1189-1205.

[13] Srinivasa Rao, R., Siva Prasad, K. and Srinivas, T., Hereditary right Jacobson radical of type-0(e) for right near-rings, Beitr. Algebra Geom., 50 (2009), no. 1, 11-23.

[14] Veldsman, S., Modulo-constant ideal-hereditary radicals of near-rings, Quaest. Math. 11 (1988), 253-278.

[15] Veldsman, S., The general radical theory of near-rings - answers to some open probems, Algebra Universalis 36 (1996), 185-189.

Department of Mathematics,

R. V. R. \& J. C. College of Engineering,

Chandramoulipuram, Chowdavaram

Guntur-522019, Andhra Pradesh, India.

Email: dr_rsrao@yahoo.com

Department of Mathematics,

Acharya Nagarjuna University,

Nagarjunanagar-522510,

Guntur (Dist.), Andhra Pradesh, India.

Email: siva235prasad@yahoo.co.in 